\title{
EARLY RESULTS FROM THE HAO/NSO ADVANCED STOKES POLARIMETER
}

B. W. LITES, D. F. ELMORE, S. TOMCZYK, P. SEAGRAVES, A. SKUMANICH, K. V. STREANDER, High Altitude Observatory, National Center for Atmospheric Research, ${ }^{1}$ P.O. Box 3000, Boulder, CO 80307, USA

\begin{abstract}
The Advanced Stokes Polarimeter is a new instrument dedicated to quantitative measurement of vector magnetic fields in lines formed at several heights in the solar atmosphere at high angular resolution. We present results for a small, symmetric sunspot observed under good seeing conditions near disk center on 25 March 1992. The narrow penumbral "spines" of more vertical field reported by Degenhardt and Wiehr (1991) and Title et al. (1992) are clearly seen in these observations. These spines are characterized by higher field strength than the surrounding penumbra. The inferred field azimuth indicates that these spines flare out with height, as would be expected of stronger field intrusions within the background penumbral field. The observations indicate the penumbral field "canopy" extends well beyond the outer edge of the penumbra. Outside of the sunspot, the small flux elements of mixed polarity are nearly vertical in orientation.
\end{abstract}

\section{THE INSTRUMENT}

As a joint project of the High Altitude Observatory (HAO) and the National Solar Observatory (NSO), the Advanced Stokes Polarimeter (ASP) integrates a number of systems of the Vacuum Tower Telescope (VTT) at NSO/Sacramento Peak with polarization optics and electronics developed by HAO. The system is described in detail by Elmore et al. (1992). Table I lists the typical operating parameters for ASP.

\section{VECTOR MAGNETIC FIELD STRUCTURE OF A SUNSPOT}

We report on ASP measurements of a small sunspot (outer penumbral radius of $10 \mathrm{Mm}$ ) observed on 1992 March 25 at 14:31UT when it was only $3.8^{\circ}$ from disk center $(\mu=0.9977$, NOAA Active Region No. 7111). Figure 1 illustrates a few of the properties of this sunspot derived from simultaneous least squares fitting of the full Stokes profiles of Fe I 630.15 and $630.25 \mathrm{~nm}$. The analysis method has been described extensively elsewhere (Lites and Skumanich 1985; Skumanich and Lites 1987; Lites and Skumanich 1990; Lites et al. 1991).

1 The National Center for Atmospheric Research is sponsored by the National Science Foundation. 
TABLE I ASP Operating Performance

\begin{tabular}{ll}
\hline Spectral sampling: & $1.271 \mathrm{pm} /$ pixel at $630 \mathrm{~nm}$, \\
& $1.029 \mathrm{pm} /$ pixel at $517 \mathrm{~nm}$ \\
Wavelength Coverage: & $0.31 \mathrm{~nm}$ at $630 \mathrm{~nm}$, \\
& $2 \times 0.120 \mathrm{~nm}$ at $517 \mathrm{~nm}$ \\
Measured Spectral Resolution: & FWHM $<3 \mathrm{pm}$ at $630 \mathrm{~nm}$ \\
$\begin{array}{l}\text { Noise Level (continuum, disk center): } \\
\text { Residual Polarization Crosstalk }\end{array}$ & $0.001 I_{c}$ \\
$\quad(V \rightarrow Q, U):$ & typically $1-2 \times 10^{-3} I_{c}$ \\
Spatial Sampling Along Slit: & $0.37 "$ per pixel \\
Spectrograph Spatial Step: & variable, multiples of $0.125^{\prime \prime}$ \\
Integration Time Per Spectral & \\
$\quad$ Measurement: & 4.1 seconds \\
$\begin{array}{l}\text { Typical Active Region Map Duration } \\
\quad\left(85 \times 85^{\prime \prime}, 0.37^{\prime \prime} \text { sampling): }\right.\end{array}$ & 16 minutes \\
\hline
\end{tabular}

The middle panel of Figure 1 demonstrates the quality of the spectropolarimetric observations possible with ASP under good seeing conditions. It is reconstructed from the measured continuum Stokes $I$ intensity at each of the slit positions of this spatial map. To the left and right are, respectively, the inferred total field strength and the field inclination with respect to the solar surface. We identify the following features of these images which describe the fine structure of the vector magnetic field:

- Fine structure in the total field strength: There is considerable structure in the strength of the magnetic field both in the umbra and penumbra of the sunspot. The distinct darker umbral areas correspond to higher field strength, as has been reported recently by Kopp and Rabin (1992), Martínez Pillet, and Vázquez (1992), Lites et al. (1991). The penumbra is characterized by nearly radial spines of higher field strength.

- Fine structure in the field inclination: The darker structures in the umbra and the umbral protrusions into the penumbra are associated with magnetic fields that are more vertically oriented. Also, within the penumbral region and extending beyond the continuum penumbral boundary, one sees many radial spines of more vertically oriented magnetic field. These spines correspond to the same features of higher field strength noted above.

- Fine structure of the field azimuth in the penumbra: Not shown here, but also clearly visible is a rotation of the field azimuth (the angular orientation of the field in the horizontal plane) across these spines, in the sense that the field is diverging away from the center line of each spine.

- Penumbral canopy: The penumbral magnetic field clearly extends beyond the outer penumbral boundary defined by the continuum intensity.

- Flux tubes outside the sunspot: Flux elements outside the sunspot are inferred to be oriented vertically with respect to the solar surface, with either positive (white) or negative (black) magnetic polarity. The inversion yields typical values for the strength of the magnetic field of the order of 1000 Gauss. 


\section{STATUS OF OBSERVATIONS AND DATA REDUCTION}

A large number of maps of active regions have already been obtained by ASP during the March and June 1992 observing runs. We have simultaneous data in both the $630 \mathrm{~nm}$ Fe I lines and the $517 \mathrm{~nm} \mathrm{Mg}$ I lines. We are also using observations of the Fe II $614.9 \mathrm{~nm}$ line to refine our polarization calibration at $630 \mathrm{~nm}$ (see Lites 1992). Much of our effort during the past few months has been directed toward refinement of this calibration. The data from the paired detectors will also be combined to improve the signal/noise ratio and to reduce residual crosstalk due to seeing fluctuations. Software for processing and management of the large ASP database is being refined.
Field Strength
(0 - 2500 Gauss)
Continuum
Spectroheliogram
Field Inclination
(Bright to Observer)
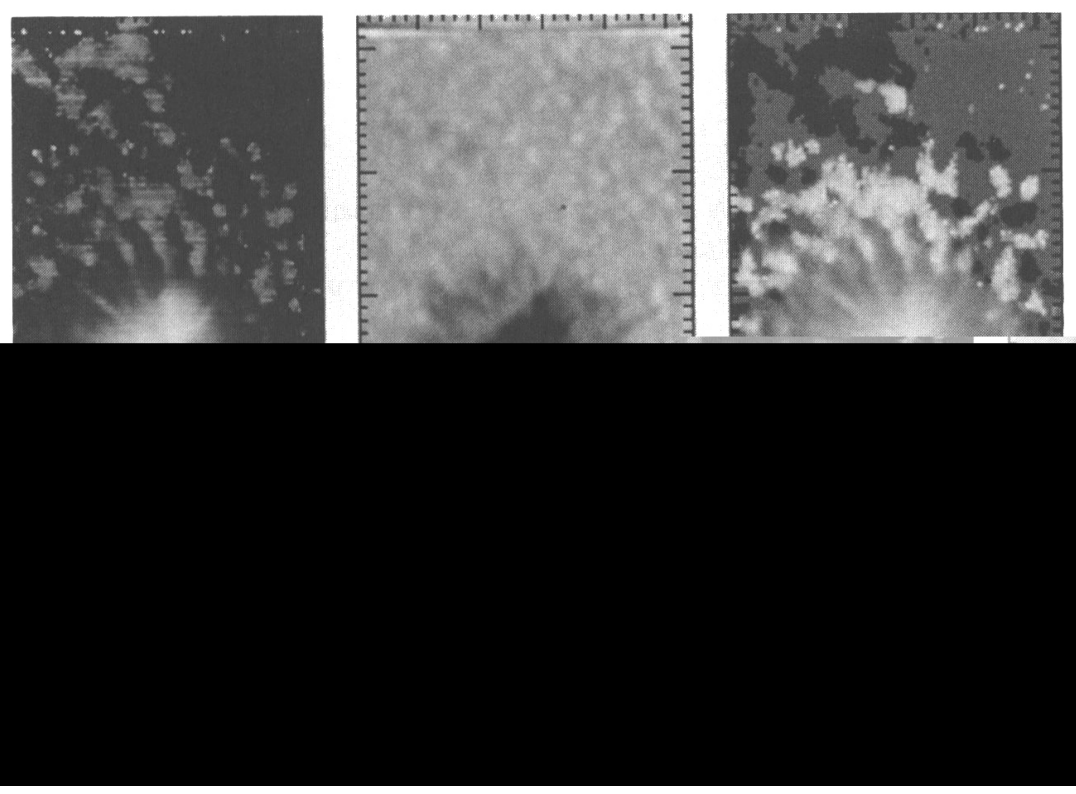

Fig. 1. The least-squares inversion of the full Stokes profiles of Fe I 630.15 and $630.25 \mathrm{~nm}$ yields the spatial variation of the vector magnetic field within and surrounding the sunspot. No fit was attempted for areas where the net polarization $<0.4 \%$. These regions are represented by uniform black in the left (total field strength) panel and gray in the right panel (field inclination). The middle panel gives the spectroheliogram of continuum intensity. Each tick mark equals 1.0 megameter. 
The cameras have been reworked to reduce or eliminate remaining problems of drift and charge transfer. The new cameras will be deployed during an observing run in October-November 1992. It is expected that the ASP will become available as a user instrument to the community in mid-1993.

\section{ACKNOWLEDGMENTS}

We thank the National Solar Observatory/Sacramento Peak and R. Smartt for undaunting support during the deployment of this instrument and during acquisition of the data. In particular, we thank Dick Dunn for all of his help and advice, especially with regard to the optical layout of the spectrograph and its feed optics. Special thanks are also due to the NSO/Sunspot Tower observers S. Hegwer, R. Mann, and E. Stratton, as well as the Tower programmer F. Stauffer for assistance throughout this program.

\section{REFERENCES}

Degenhardt, D. and Wiehr, E. 1991, Astr. Ap., 252, 281.

Elmore, D. F., Lites, B. W., Tomczyk, S., Skumanich, A. P., Dunn, R. B., Schuenke, J. A., Streander, K. V., Leach, T. W., Chambellan, C. W., Hull, H. K., and Lacey, L. B. 1992, in SPIE Proceedings, submitted.

Kopp, G. and Rabin, D. 1992, Solar Phys. 141, 253.

Lites, B. W. 1992, Solar Phys, in press.

Lites, B. W., Bida, T. A., Johannesson, A., and Scharmer, G. B. 1991, Ap. J., 373,683 .

Lites, B. W. and Skumanich, A. 1985, in Measurements of Solar Vector Magnetic Fields, ed. M Hagyard (NASA Marshall Space Flight Center), 342. 1990, Ap. J., 348, 747.

Martínez Pillet, V. and Vázquez, M. 1992, Astr. Ap., in press.

Skumanich, A. and Lites, B. W. 1987, Ap. J., 322, 473.

Title, A. M., Frank, Z. A., Shine, R. A., Tarbell, T. D., Topka, K. P., Scharmer, G. B., and Schmidt, W. 1992, in Sunspots: Theory and Observations, NATO ASI series, ed. J. H. Thomas and N. O. Weiss (Dordrecht: Kluwer), 375. 